\title{
Evaluation and management of toxicity of cytoreductive surgery/hyperthermic intraperitoneal chemotherapy: the initial experience of a single centre study
}

\author{
Radmila Lemstrovaa, Dominika Flasarova ${ }^{a}$, Martina Spisarovaa , Bohuslav Melichara, ${ }^{\mathrm{a},}$, Martin Lovecek, Roman Havlikc, \\ Cestmir Neoral', Beatrice Mohelnikova-Duchonova ${ }^{a}$, Dusan Klos ${ }^{b}$
}

\begin{abstract}
Background. Cytoreductive surgery (CRS) and hyperthermic intraperitoneal chemotherapy (HIPEC) is a treatment modality for peritoneal surface malignancies with efficacy reported in many trials. Discrepancies, however, in the indication criteria, the extent of the surgical procedure, HIPEC regimens and toxicity evaluation represent a problem when comparing this method with other therapeutic modalities.

Methods. We describe the initial experience with CRS/HIPEC using different chemotherapy regimens (oxaliplatin, cisplatin, mitomycin $\mathrm{C}$ and doxorubicin) at the Comprehensive Oncology Centre Olomouc.

Results. A perioperative mortality of $2 \%$ and perioperative morbidity of $11 \%$, according to Clavien-Dindo were observed. Interestingly, all these patients underwent HIPEC with oxaliplatin $460 \mathrm{mg} / \mathrm{m}^{2}$. The median duration of admission to hospital was 6 days in the intensive care unit (range 2-28 days) and 7 days in the surgical ward (range 1-21 days). Hospital admission did not exceed 2 weeks in $75 \%$ of patients. These results are consistent with the published results of large centres performing this treatment modality mainly due to pre-operative preparation of patients and pre-treatment and post-treatment management of HIPEC/CRS toxicity. Evaluation of the efficacy in terms of time to progression and overall survival (OS) is limited by the short follow up period.
\end{abstract}

Conclusion. CRS/HIPEC performed is a safe method with low perioperative mortality.

Key words: cytoreductive surgery, hyperthermic intraperitoneal chemotherapy, oxaliplatin, mitomycin C, cisplatin, toxicity, adverse events, morbidity

Received: February 27, 2019; Revised: July 10, 2019; Accepted: July 22, 2019; Available online: August 21, 2019 https://doi.org/10.5507/bp.2019.035

(c) 2020 The Authors; https://creativecommons.org/licenses/by/4.0/

${ }^{a}$ Department of Oncology, Faculty of Medicine and Dentistry, Palacky University Olomouc and University Hospital Olomouc, Czech Republic ' Institute of Molecular and Translational Medicine, Faculty of Medicine and Dentistry, Palacky University Olomouc, Czech Republic 'Department of Surgery l, Faculty of Medicine and Dentistry, Palacky University Olomouc and University Hospital Olomouc, Czech Republic Corresponding author: Dusan Klos, e-mail: dusan.klos@fnol.cz

\section{INTRODUCTION}

Surgery, radiation and pharmacological therapy represent the three principal components of the multimodality curative treatment of cancer. Cytoreductive surgery (CRS)/ hyperthermic intraperitoneal chemotherapy (HIPEC), a therapeutic method consisting of radical surgery, regional application of cytotoxic agents and hyperthermia, is unique in combining all of these three fundamental approaches in the treatment of peritoneal malignancies. In an era of targeted therapy, CRS/HIPEC represents an alternative concept to molecular targeting, i.e. anatomical targeting of anticancer drugs, increasing the efficacy by potential synergisms with surgical cytoreduction and with the effect of hyperthermia. The options for the treatment of peritoneal carcinomatosis were limited until the advent of CRS/HIPEC. Peritoneal carcinomatosis is considered the terminal stage of a disease. The efficacy of systemic chemotherapy is limited by poor vascularisation of the peritoneum ${ }^{1}$, the presence of tumour hypertension, which prevents the influx of cytotoxic drugs into the tumour tissue ${ }^{2}$, and also by the fact that most tumors presenting with peritoneal carcinomatosis are only moderately sensitive to cytotoxic drugs. The incidence of peritoneal malignancies is not negligible. The incidence ranges from $0.2-3$ cases per million people per year, for rare tumours such as mesothelioma, to 4 to 7.2 cases per 100000 people per year for secondary peritoneal malignancies such as colorectal cancer or ovarian cancer ${ }^{3}$.

CRS/HIPEC combines extensive surgical procedure with administration of heated $\left(41-43{ }^{\circ} \mathrm{C}\right)$ high dose chemotherapy. The surgical procedure includes peritoneal surface stripping, omental resection, multiple visceral resections (such as splenectomy, appendectomy, and bowel resection) and lymphadenectomy of regional basins. Mitomycin C, cisplatin, oxaliplatin, doxorubicin and paclitaxel are the cytotoxic agents most commonly used in HIPEC protocols. The duration of the surgery is around 10 h. Nevertheless, low mortality from 0.9 to $3.2 \%$ and acceptable morbidity of $12-33 \%$ is reported when appropriate procedure and selection of patients is performed in tertiary centres ${ }^{4}$. There is no unified classification system of treatment toxicity. Different authors use several classification systems including Clavien-Dindo or Common Terminology Criteria of Adverse Events (CTCAE) v3.0. The reported serious toxicity differs according to the in- 
dividual classification system from $11 \%$ to $60 \%$ (ref. $^{4-6}$ ) and declines within 4 months after CRS/HIPEC ( ref. $^{7}$ ).

The efficacy of CRS/HIPEC in the treatment of peritoneal malignancies has been demonstrated in a number of prospective trials. CRS/HIPEC have been reported to prolong the median OS to 5 years, - i.e. by $50 \%$ compared with conventional surgery in the treatment of pseudomyxoma peritonei. The median OS of patients with mesothelioma peritonei has been reported to increase from 12 months to 34-92 months ${ }^{8,9}$. The results of the randomized phase 3 trial, comparing CRS with CRS/HIPEC in the treatment of inoperable ovarian cancer stage FIGO III, revealed the prolongation of median OS by 11.8 months in favour of CRS + HIPEC (ref. ${ }^{10}$ ). The efficacy of CRS/ HIPEC has also been demonstrated in the treatment of peritoneal carcinomatosis of colorectal origin, a disease entity with a dismal prognosis. CRS/HIPEC has increased the median OS by 12 months compared with standard systemic chemotherapy ${ }^{11}$. There are several ongoing randomized trials evaluating the efficacy of CRS/HIPEC in the treatment of peritoneal carcinomatosis of gastric primary.

In spite of these encouraging results, CRS/ HIPEC has yet not become the standard of care. There is a lack of a unified system for adverse events classification and evaluation of peritoneal surface involvement. Moreover, there is a difference in the extent of the surgical procedure, the method of the lavage as well as in the HIPEC regimens. The choice of the appropriate cytotoxic agents or drug combination is crucial. The cytotoxic agents should not lead to local intraperitoneal toxicity. Agents which are systemically metabolized into an active form are also not suitable. There is an important synergism between the drug and hyperthermia. Heat influences chemotherapy cytotoxicity and the pharmacokinetics in terms of influx and efflux of the agent into the tissue.

We present the initial experience and preliminary results of CRS/HIPEC performed in the Comprehensive Oncology Centre Olomouc with evaluation and management of CRS/HIPEC toxicity.

\section{PATIENTS AND METHODS}

We retrospectively evaluated a cohort of 44 patients who underwent CRS/ HIPEC between 1 January 2016 and 1 January 2018 in the Comprehensive Oncology Centre Olomouc. The clinical and pathologic parameters of all the patients, such as gender, age, site of the primary, extent of the disease, extent of the peritoneal involvement (evaluated with peritoneal cancer index; PCI), extent and completeness of the surgical procedure (evaluated with the CCS score), previous surgical and systemic treatment, postoperative systemic treatment and time to progression of the disease (progression-free survival; PFS) were retrieved. PFS was defined as the time from the date of the CRS/HIPEC procedure to the date of progression of the disease. The date of progression was based on clinical and imaging examination. Tumour markers (CA 125, CEA, CA 19-9, and CA 72-4) were examined in 3-month intervals. Computed Tomography scan of the thorax, abdomi- nal cavity and pelvis were performed in 6-month intervals. The Kaplan-Meier method was used to calculate median PFS with the help of software SAS EG 5.

The adverse events, toxicity and safety of the treatment have been evaluated according to the surgical classification Clavien-Dindo (postoperative morbidity), length of hospital admission, stay in the intensive care unit and in the surgical ward. Perioperative mortality was defined as the ratio of the deceased patients, within 30 days of the procedure to the total number of the patients who underwent CRS/HIPEC.

All patients were discussed by a multidisciplinary team.

The indication criteria were as follows:

$1 /$ disease disseminating to the peritoneum

2/ no more effective treatment option available

3/ performance status of patients PS 0-1 according to the WHO classification

The contraindications were:

1/ the presence of visceral metastases, e.g. liver metastases, pulmonary metastases; or the presence of bone metastases

2/ the performance status of patients PS 2 and less according to the WHO classification

3/ surgical contraindications including involvement of large veins of arteries, metastases of small intestine serosa and invasion of the visceral organs or the abdominal wall

4/ comorbidities contraindicating large surgical procedures

Eligible patients for CRS/HIPEC signed an informed consent with the procedure. Regarding the safety of the

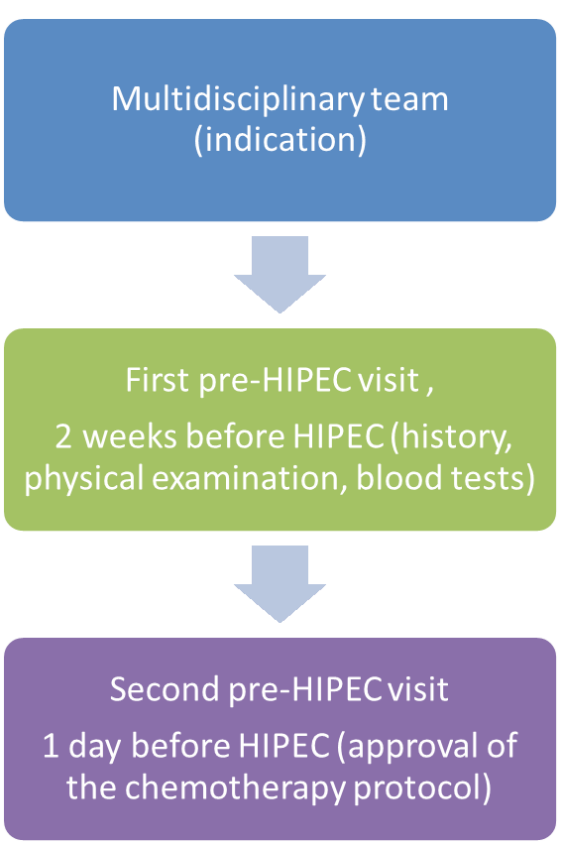

Fig. 1. Scheme of pre-HIPEC preparation of patients. 
Table 1. Subgroups of patients according to the primary tumor.

\begin{tabular}{lcccccc}
\hline Primary & $\mathrm{n}$ & Recurrent disease / Primary treatment & PCI (range) & CCS0 & CCS1 & CCS2 \\
\hline Ovarian cancer & 17 & $7 / 10$ & $5(2-39)$ & 10 & 3 & 4 \\
Colorectal cancer & 9 & $8 / 1$ & $10(1-25)$ & 6 & 0 & 3 \\
Pseudomyxoma peritonei & 8 & $1 / 7$ & $15(6-34)$ & 6 & 1 & 1 \\
Mesothelioma & 9 & $0 / 9$ & $20(3-31)$ & 5 & 2 & 2 \\
Endometrial cancer & 1 & $1 / 0$ & 6 & 0 & 0 & 1 \\
\hline
\end{tabular}

n - number of patients, PCI - Peritoneal cancer index, CCS - cytoreduction score defined as macroscopically complete surgery (CCS0); optimal residual disease $\leq 2.5 \mathrm{~mm}$ in any region (CCS-1); or largely incomplete surgery with residual disease $>2.5 \mathrm{~mm}(\mathrm{CCS}-2)$.

procedure, patients are followed in several pre HIPEC and post HIPEC visits (Fig. 1).

Special attention was paid to nutritional status, risk of infection, medication especially regarding corticosteroids and oral anticoagulant use which may affect postoperative complications such as bleeding, delayed surgical wounds healing, pressure lesions, systemic infections, respiratory failure and increased hospitalization duration and increased mortality rate r2-14 $^{12}$

The first visit took place 2 weeks before CRS/HIPEC with a review of the patient's history, changes in medication, physical examination and complete blood test. The second visit followed one day before CRS/HIPEC with actual blood tests and a physical examination. The HIPEC regimen protocol is only approved in case of no contraindications. The post-CRS/ HIPEC visit to assess additional toxicity and manage adverse events followed 4 to 6 weeks after the procedure. The patients are followed further in 3 month intervals.

\section{RESULTS}

Forty-four patients, 32 females and 12 males, were included in the retrospective analysis. All patients underwent the close method of CRS/HIPEC procedure. The cohort consisted of 17 patients with ovarian cancer, 9 patients with colorectal cancer, 9 patients with peritoneal mesothelioma, 8 patients with pseudomyxoma peritonei and 1 patient with endometrial cancer. The median age of the patients was 61 years (range 36 to 76 years).

The subgroup of 17 patients with ovarian cancer included 7 patients with the second or third recurrence of the disease. Ten patients had prior surgery for ovarian cancer. The median PCI was 5 (range 2-39). Cisplatin $\left(75 \mathrm{mg} / \mathrm{m}^{2}\right)$ was administered in 16 patients and mitomycin $\mathrm{C}\left(30 \mathrm{mg} / \mathrm{m}^{2}\right)$ was administered in one patient with platinum-resistant disease. Ten patients had complete cytoreduction CCS0, 3 patients CCS1 and 4 patients CCS2 (Tables 1 and 2).

Only one patient out of 9 in the subgroup with colorectal cancer had no prior treatment. All the other patients had recurrent disease and had prior surgical or systemic treatment. The median PCI was 10 (range 1-25). Oxaliplatin $\left(460 \mathrm{mg} / \mathrm{m}^{2}\right)$ was administered to 7 patients and mitomycin $\mathrm{C}\left(30 \mathrm{mg} / \mathrm{m}^{2}\right)$ was used with 2 patients. Six patients underwent complete cytoreduction CCSO. It
Table 2. HIPEC regimens used in COC Olomouc.

\begin{tabular}{lll}
\hline Primary & HIPEC regimen & $\mathrm{n}$ \\
\hline Colorectal cancer & oxaliplatin $460 \mathrm{mg} / \mathrm{m}^{2}$ & 7 \\
mitomycin C $30 \mathrm{mg} / / \mathrm{m}^{2}$ & 2 \\
Mesothelioma peritonei & $\begin{array}{l}\text { cisplatin } 50 \mathrm{mg} / \mathrm{m}^{2} \\
\text { doxorubicin } 15 \mathrm{mg} / \mathrm{m}^{2}\end{array}$ & 9 \\
Ovarian cancer & cisplatin $75 \mathrm{mg} / \mathrm{m}^{2}$ & 17 \\
& mitomycin C $30 \mathrm{mg} / \mathrm{m}^{2}$ & 1 \\
Pseudomyxoma peritonei & Oxaliplatin $460 \mathrm{mg} / \mathrm{m}^{2}$ & 7 \\
& mitomycin C $30 \mathrm{mg} / \mathrm{m}^{2}$ & 1 \\
Endometrial cancer & cisplatin $75 \mathrm{mg} / \mathrm{m}^{2}$ & 1 \\
\hline
\end{tabular}

$\mathrm{n}$ - number of patients.

was impossible to accomplish a complete cytoreduction with 3 patients; the score of the cytoreduction was CCS 2.

With one exception, all the patients in the subgroup with pseudomyxoma peritonei underwent CRS/HIPEC as the primary treatment. Only one patient had a recurrent disease. The median PCI was 15 (range 6-34). Oxaliplatin $\left(460 \mathrm{mg} / \mathrm{m}^{2}\right)$ was administered in 8 patients, and mitomycin $\mathrm{C}\left(30 \mathrm{mg} / \mathrm{m}^{2}\right)$ was administered in one patient. Complete cytoreduction of CCS 0 was achieved in 6 cases, one patient had minimal residual disease CCS1, and one patient had incomplete cytoreduction of CCS 2 .

All the patients with mesothelioma peritonei were treatment-naive. A definitive histology confirmed sarcomatoid mesothelioma in one patient while other patients had epithelial peritoneal mesothelioma. The median PCI was 20 (range 3-31). All the patients were treated with cisplatin $\left(50 \mathrm{mg} / \mathrm{m}^{2}\right)$ and doxorubicin $\left(15 \mathrm{mg} / \mathrm{m}^{2}\right)$. Optimal cytoreduction of CCS- 0 was achieved with 5 patients, cytoreduction of CCS- 1 with 2 patients, and CCS- 2 was accomplished in with 2 patients.

\section{Preliminary results of PFS in the subgroups of patients}

The median PFS has not been reached in the subgroup of 17 patients with ovarian cancer. The median of the follow up was 7 months (range 1-19 months; Fig. 2). In the subgroup of patients with colorectal cancer, the median PFS was 6 months (95\%CI: 3-6.3 months; Fig. 3). No patient with pseudomyxoma peritonei has progressed during the median follow up of 7 months (range 1-16 months). One patient with mesothelioma peritonei had a disease 


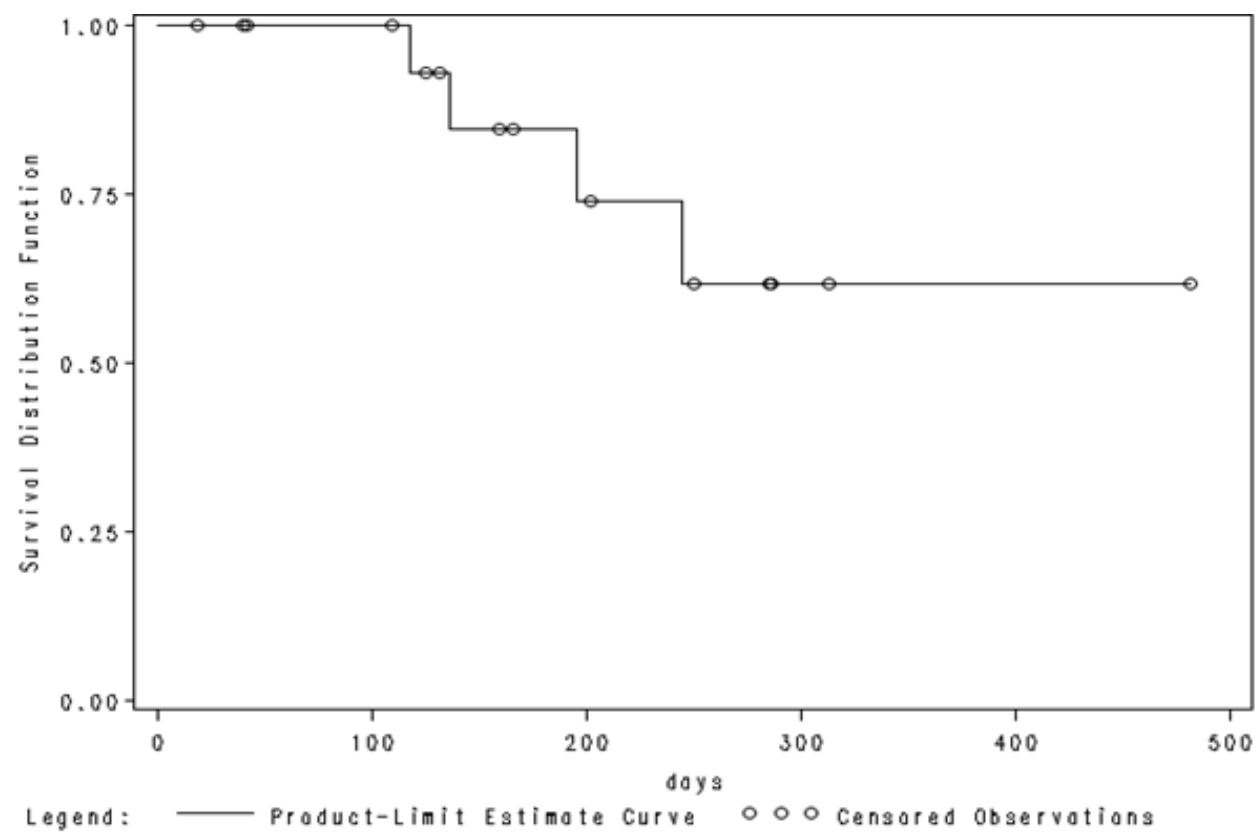

Fig. 2. Progression-free survival of patients with ovarian cancer.

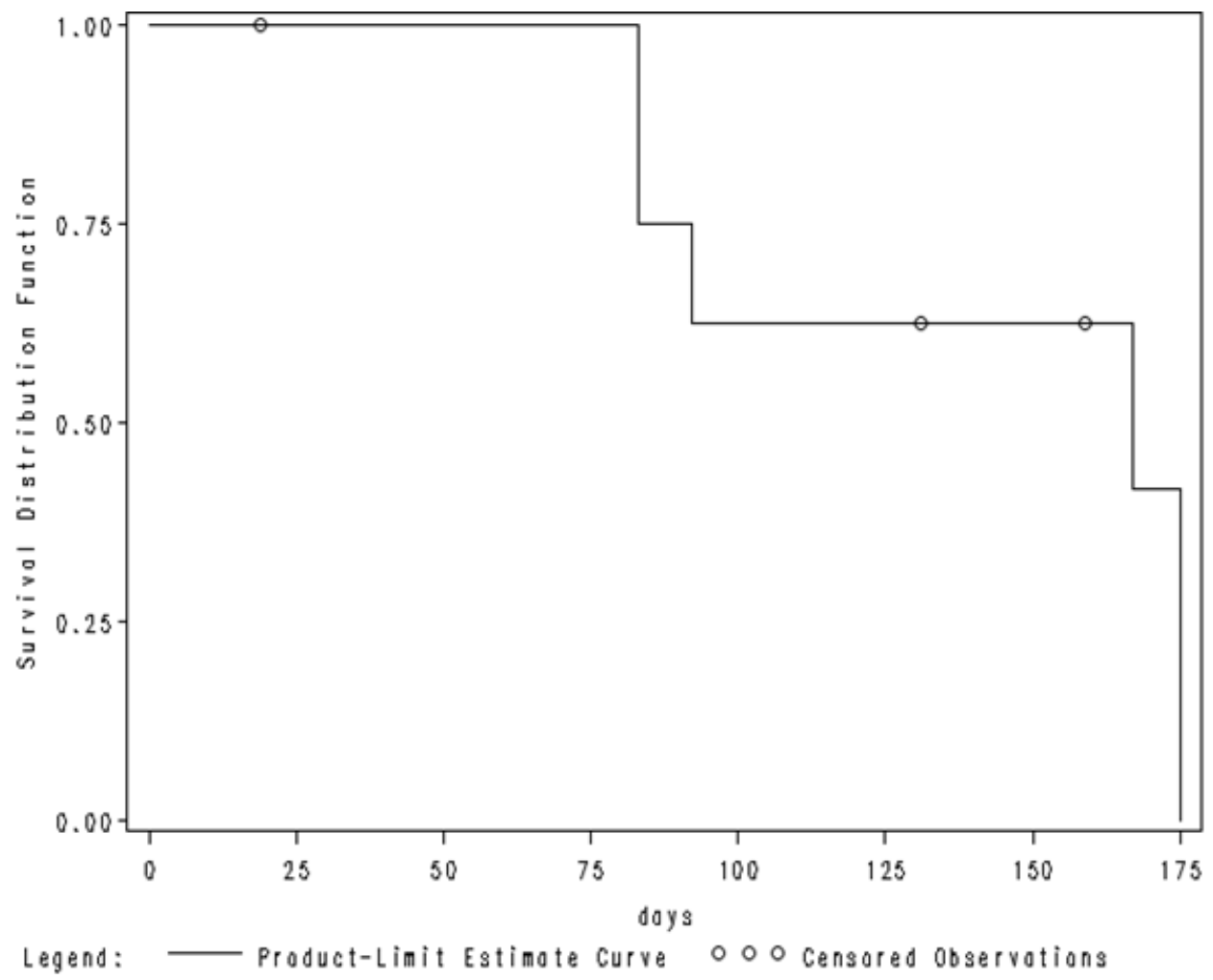

Fig. 3. Progression-free survival of patients with colorectal cancer.

progression, and the median follow up in this subgroup was 10 months (range 1-22 months). One patient with endometrial cancer was free of the disease 9 months after CRS/ HIPEC.

\section{Adverse events, toxicity and safety of the treatment}

Perioperative mortality was $2 \%$ (1/44) due to acute liver necrosis in one patient who received HIPEC oxaliplatin during the first week after surgery. Serious perioperative morbidity grade 3 and 4, according to the Clavien-Dindo classification, was $11 \%$ (5/44). Serious complications occurred in 4 patients with colorectal cancer and 1 patient with pseudomyxoma peritonei. All these patients underwent HIPEC with oxaliplatin $460 \mathrm{mg} / \mathrm{m} 2$. The patients were re-operated for suspected abdominal bleeding and inflammatory complications. 
We observed postoperative non-surgical bleeding in the peritoneal cavity in our set with oxaliplatin HIPEC administration. We have not found any surgical source, eg. bleeding from vessels or from visceral organs as liver or spleen. This was a kind of diffuse bleeding from the soft tissues, from the retroperitoneum and abdominal wall after peritonectomy even if we have used a very precise surgical technique with the use of advanced thermocoagulation.

The median duration of admission to hospital was 6 days in the intensive care unit (range 2-28 days) and 7 days in the surgical ward (range 1-21 days). The hospital admission did not exceed 2 weeks in $75 \%$ of the patients.

\section{DISCUSSION}

The results of the present cohort of patients show a low perioperative mortality and acceptable morbidity associated with CRS/HIPEC with the proposed management of pre-HIPEC and post-HIPEC visits. No prolonged admission to either the intensive care unit or general surgical ward was reported. Most patients were discharged within 14 days of the procedure with the possibility of sequential adjuvant systemic treatment. The results of perioperative mortality of $2 \%$ and postoperative morbidity of $11 \%$ are consistent with the published results of high volume centres ${ }^{4-6}$. The results reflect the experience of the centre in terms of the "learning curve" and correct patient selection by the multidisciplinary team ${ }^{15}$. The perioperative mortality and morbidity are influenced by the extent of the surgical procedure, HIPEC regimens used as well as by the performance status of the patient, age, nutritional status, previous antitumor treatment, comorbidities and current medication. Increased morbidity is associated with performance status $>1$, age $>60$ years and hypoalbuminemia. Additional complications occur in obese patients ${ }^{15}$. We believe that the scheme of pre-HIPEC and post-HIPEC visits with intervention to minimize the complications also contributed to the low morbidity and mortality numbers in our cohort. Serious morbidity was mainly reported in patients with colorectal cancer who had a recurrent disease and were mostly heavily pre-treated. In patients with colorectal cancer, the median PCI was 10 and complete cytoreduction was accomplished only in 50\%. The HIPEC regimen utilized was oxaliplatin at a dose of $460 \mathrm{mg} / \mathrm{m}^{2}$ administered in $30 \mathrm{~min}$. Oxaliplatin is one of the most commonly used cytotoxic agents in HIPEC regimens. Oxapliplatin efficacy and toxicity have been evaluated in several retrospective trials. Some authors report the superiority of oxaliplatin above mitomycin $\mathrm{C}$ in terms of prolonged OS (ref. ${ }^{16}$ ), mostly in tumours with an unfavorable histology and more extensive involvement of peritoneum ${ }^{17}$. The present results differ from previously published retrospective data that did not report an increased rate of oxaliplatin toxicity compared to other cytotoxic agents ${ }^{18-20}$ but are consistent with the results of the large randomized trial Prodige 7 $\left(\right.$ ref. $\left.^{21}\right)$. This trial did not show greater efficacy of HIPEC with oxaliplatin in terms of prolonged OS or PFS compared to cytoreductive surgery alone, but increased risk of postoperative complications. The potential benefit of oxaliplatin was only observed in patients with low PCI from 11 to 15 , and in this subgroup of patients the median OS was prolonged compared to surgery alone ${ }^{21}$.

The evaluation of time to progression is limited by the short duration of the follow up in the present cohort. The cohort is also very heterogeneous even when split into subgroups of patients according the primary site. The heterogeneity with regard to the prior treatment of the patients and recurrence of the disease is mainly pronounced in the subgroup of patients with ovarian cancer and colorectal cancer. There is also heterogeneity in the HIPEC regimens and completeness of surgery. Only one patient had progression of the disease in the subgroups with mesothelioma and pseudomyxoma peritonei in the follow up of 7 to 10 months. These results are consistent with the published data that consider CRS/HIPEC as a standard therapeutic modality in this setting. In the case of pseudomyxoma peritonei, CRS/HIPEC has a prolonged median PFS to 8.2 years and median OS to 16.3 years $^{22}$. In case of mesothelioma peritonei, conventional surgery and systemic chemotherapy resulted in a median OS of 1 year whereas CRS/HIPEC led to a median OS of 2.8 to 7.8 years $^{23}$.

The results of a prospective randomized trial of ovarian cancer, comparing CRS versus CRS/HIPEC, confirm CRS/HIPEC as a standard treatment modality in ovarian cancer stage FIGO III. This trial reported a median PFS of 14.2 months in a patient treated with CRS/ HIPEC versus 10 months in patients treated with CRS alone. The median OS was prolonged by 11 months ${ }^{10}$. The present cohort included 7 patients with recurrent ovarian cancer. In case of recurrent ovarian cancer, a much shorter PFS is expected. The PFS in a randomized phase 3 trial of recurrent ovarian cancer and maintenance therapy with PARP inhibitors reached only 5.5 months in the placebo group ${ }^{24}$. In the subgroup of patients with ovarian cancer the median PFS was not reached after a follow up of 7 months.

The prognosis of carcinomatosis of colorectal origin is dismal, with the reported median OS ranging between 5-12 months ${ }^{25}$. CRS/HIPEC initially brought some encouraging results which were presented by Verwaal et al. ${ }^{26}$. In the randomized trial, CRS/HIPEC prolonged OS by 5 months compared to systemic treatment of the peritoneal carcinomatosis. Similar results were reported by Elias et al. ${ }^{27}$ in the patients treated with CRS/HIPEC the median OS was 63 months. These results were not confirmed, however, by prospective randomized trial PRODIGE 7 presented by Quenet et al. ${ }^{21}$. The trial compared CRS versus CRS/HIPEC. Patients who underwent CRS/HIPEC with oxapliplatin had more postoperative complications. The key role of cytoreductive surgery was emphasized, however, in this study. The median PFS in the subgroup of patients with colorectal cancer in the present cohort reached only 6.2 months. As mentioned previously, however, this subgroup consisted of heavily pre-treated 
patients, and only $50 \%$ of the patients had complete cytoreduction of CCSO.

This initial experience and preliminary results confirm the safety of the combined treatment modality of CRS/ HIPEC although the results are limited by the size and heterogeneity of the cohort. More complications were seen in HIPEC with oxaliplatin in the present cohort. These results are consistent with the recently published data from a prospective randomized trial of colorectal cancer and differ from previous retrospective trials. A larger cohort of patients is needed to compare the toxicity of the individual HIPEC regimens.

\section{CONCLUSION}

Correct indication of the patients by multidisciplinary teams is essential. Close follow up with pre-HIPEC and post-HIPEC visits with early interventions may contribute to low morbidity and mortality numbers. The performance status of the patients, previous treatments and the possibility of performing complete cytoreduction of CCS0-1 needs to be taken into consideration. The present preliminary results need to be assessed with caution with regard to the short duration of the follow up and heterogeneity of the cohort. However, these results support the benefit of CRS/HIPEC in the treatment of patients with pseudomyxoma peritonei, peritoneal mesothelioma and ovarian cancer.

Acknowledgment: This work was supported by grant project AZV MZČR NV18-03-00130 to D.K., student grant project IGA_LF_2018_010 from Palacky University to D.F., and Department for Administration and Development project, Ministry of Education, Youth and Sport, Czech Republic, Molecular and Cellular Clinical Approach to Healthy Ageing (ENOCH project) via IMTM to B.M.

Author contributions: RL, DF, MS, DK: literature search; BMD, BM, RH, DK: conceptualization; RL, CN, DK, ML, BMD: methodology; DK, VMD, BM, RL: validation; RL, DF, MS: data processing; DK, RL, BMD: data analysis; RL, DF, ML, DK: manuscript writing- original draft preparation; BM, BMD: writing- review and editing; RH, BM: supervising, CN, DK: project administration; DK, BMD: funding acquisition; BMD, DK: final approval. Conflicts of interest statement: The authors state that there are no conflicts of interest regarding the publication of this article.

\section{REFERENCES}

1. Markman M. Intraperitoneal antineoplastic drug delivery: rationale and results. Lancet Oncol 2003;4(5):277-83.

2. Minchinton Al, Tannock IF. Drug penetration in solid tumours. Nat Rev Cancer 2006;6(8):583.

3. The national oncoloy registry, www.uzis.cz/registry-nzis/nor

4. Kusamura S, Younan R, Baratti D, Costanzo P, Favaro M, Gavazzi C, Deraco M. Cytoreductive surgery followed by intraperitoneal hyperthermic perfusion: analysis of morbidity and mortality in 209 perito- neal surface malignancies treated with closed abdomen technique. Cancer 2006;106(5):1144-53.

5. Haslinger M, Francescutti V, Attwood K, McCart JA, Fakih M, Kane IIIJM, Skitzki JJ. A contemporary analysis of morbidity and outcomes in cytoreduction/hyperthermic intraperitoneal chemoperfusion. Cancer Med 2013;2(3):334-42.

6. Gusani NJ, Cho SW, Colovos C, Seo S, Franko J, Richard S D, Edwards RP, Brown CK, Holtzman MP, Zeh HJ, Bartlett DL... Aggressive surgical management of peritoneal carcinomatosis with low mortality in a high-volume tertiary cancer center. Ann Surg Oncol 2008;15(3):75463.

7. Ford J, Hanna M, Boston A, Berri R. Life after hyperthermic intraperitoneal chemotherapy; measuring quality of life and performance status after cytoreductive surgery plus hyperthermic intraperitoneal chemotherapy. Am J Surg 2016:211(3):546-50.

8. Sugarbaker PH. New standard of care for appendiceal epithelial neoplasms and pseudomyxoma peritonei syndrome? Lancet Oncol 2006;7(1):69-76.

9. Yan TD, Welch L, Black D, Sugarbaker PH. A systematic review on the efficacy of cytoreductive surgery combined with perioperative intraperitoneal chemotherapy for diffuse malignancy peritoneal mesothelioma. Ann Oncol 2006;18(5):827-34.

10. Van Driel WJ, Koole SN, Sikorska K, Schagen van Leeuwen JH, Schreuder HW, Hermans RH, et al. Hyperthermic intraperitoneal chemotherapy in ovarian cancer. N Eng J Med 2018;378(3):230-40.

11. Verwaal VJ, van Ruth $S$, de Bree $E$, van Slooten GW, van Tinteren $\mathrm{H}$, Boot $\mathrm{H}$, Zoetmulder FA. Randomized trial of cytoreduction and hyperthermic intraperitoneal chemotherapy versus systemic chemotherapy and palliative surgery in patients with peritoneal carcinomatosis of colorectal cancer. J Clin Oncol 2003;21(20):3737-43.

12. Mignini EV, Scarpellini E, Rinninella E, Lattanzi E, Valeri MV, Clementi N, Abenavoli L, Gasbarrini A, Rasetti C, Santori P.. Impact of patients nutritional status on major surgery outcome. Eur Rev Med Pharmacol Sci 2018;22(11):3524-33.

13. Subramanian V, Saxena S, Kang JY, Pollok RC. Preoperative steroid use and risk of postoperative complications in patients with inflammatory bowel disease undergoing abdominal surgery. Am J Gastroenterol 2008;103(9)2373.

14. Belli S, Aytac HO, Yabanoglu H, Karagulle E, Parlakgumus A, Nursal TZ, Yildirim S. Results of surgery in general surgical patients receiving warfarin: retrospective analysis of 61 patients. Int Surg 2015;100(2):225-32.

15. Newton AD, Bartlett EK, Karakousis GC. Cytoreductive surgery and hyperthermic intraperitoneal chemotherapy: a review of factors contributing to morbidity and mortality. J Gastrointest Oncol 2016;7(1):99-111.

16. van Eden WJ, Kok NFM, Woensdregt $K$, Huitema ADR, Boot $H$, Aalbers AGJ. Safety of intraperitoneal Mitomycin $C$ versus intraperitoneal oxaliplatin in patients with peritoneal carcinomatosis of colorectal cancer undergoing cytoreductive surgery and HIPEC. Eur J Surg Oncol 2018;44(2):220-7.

17. Leung V, Huo YR, Liauw W, Morris DL. Oxaliplatin versus Mitomycin C for HIPEC in colorectal cancer peritoneal carcinomatosis. Eur J Surg Oncol 2017;43(1):144-9.

18. Prada-Villaverde A, Esquivel J, Lowy AM, Markman M, Chua T, Pelz, Baratti D, Baumgartner JM, Berri R, Bretcha-Boix P, Deraco M, FloresAyala G, Glehen O, Gomez-Portilla A, González-Moreno S, Goodman M, Halkia E, Kusamura S, Moller M, Passot G, Pocard M, Salti G, Sardi A, Senthil M, Spiliotis J, Torres-Melero J, Turaga K, Trout R. The American Society of Peritoneal Surface Malignancies evaluation of HIPEC with Mitomycin C versus Oxaliplatin in 539 patients with colon cancer undergoing a complete cytoreductive surgery. J Surg Oncol 2014;110(7):779-85.

19. Votanopoulos K, Ihemelandu C, Shen P, Stewart J, Russell G, Levine EA. A comparison of hematologic toxicity profiles after heated intraperitoneal chemotherapy with oxaliplatin and mitomycin C. J Surg Res 2013;179(1):e133-e139.

20. Glockzin G, von Breitenbuch P, Schlitt HJ, Piso P. Treatment-related morbidity and toxicity of CRS and oxaliplatin-based HIPEC compared to a mitomycin and doxorubicin-based HIPEC protocol in patients with peritoneal carcinomatosis: a matched-pair analysis. J Surg Oncol 2103;107(6):574-8.

21. Quenet F, Elias D, Roca L, Goere D, Ghouti L, Marchal F. A UNICANCER phase III trial of hyperthermic intra-peritoneal chemotherapy 
(HIPEC) for colorectal peritoneal carcinomatosis PRODIGE 7. Eur J Surg Oncol 2019;45(2):e17.

22. Chua TC, Moran BJ, Sugarbaker PH, Levine EA, Glehen O, Gilly FN Baratti D, Deraco M, Elias D, Sardi A, Liauw W, Yan TD, Barrios P, Gómez Portilla A, de Hingh IH, Ceelen WP, Pelz JO, Piso P, González-Moreno $\mathrm{S}$, Van Der Speeten K, Morris DL.. Early-and long-term outcome data of patients with pseudomyxoma peritonei from appendiceal origin treated by a strategy of cytoreductive surgery and hyperthermic intraperitoneal chemotherapy. J Clin Oncol 2012;30(20):2449-56.

23. Yan TD, Welch $L$, Black D, Sugarbaker PH. A systematic review on the efficacy of cytoreductive surgery combined with perioperative intraperitoneal chemotherapy for diffuse malignancy peritoneal mesothelioma. Ann Oncol 2006;18(5):827-34.

24. Pujade-Lauraine E, Ledermann JA, Penson RT, Oza AM, Korach J, Huzarski T, et al. Treatment with olaparib monotherapy in the main- tenance setting significantly improves progression-free survival in patients with platinum-sensitive relapsed ovarian cancer: results from the phase III SOLO2 study. Gynecol Oncol 2017;145(suppl 1):219-20.

25. Koppe MJ, Boerman OC, Oyen WJ, Bleichrodt RP. Peritoneal carcinomatosis of colorectal origin: incidence and current treatment strategies. Ann Surg 2006;243(2):212.

26. Verwaal VJ, van Ruth $S$, de Bree E, van Slooten GW, van Tinteren $\mathrm{H}$, Boot $\mathrm{H}$, Zoetmulder FA. Randomized trial of cytoreduction and hyperthermic intraperitoneal chemotherapy versus systemic chemotherapy and palliative surgery in patients with peritoneal carcinomatosis of colorectal cancer. J Clin Oncol 2003;21(20):3737-43.

27. Elias D, Lefevre JH, Chevalier J, Brouquet A, Marchal F, Classe JM., Ferron G, Guilloit JM, Meeus P, Goéré D, Bonastre J. Complete cytoreductive surgery plus intraperitoneal chemohyperthermia with oxaliplatin for peritoneal carcinomatosis of colorectal origin. J Clin Oncol 2008;27(5):681-5. 